\title{
Correction to: Die antiklerikale Reformation und ihr Feindbild, der „Dr. Theologiae" Faustus
}

\section{Andrew Weeks ${ }^{1}$}

Published online: 27 September 2018

(c) Springer Nature B.V. 2018

\section{Correction to: Neophilologus (2018) 102:217-240 https://doi.org/10.1007/s11061-017-9550-8}

In the original publication of the article, the following symbols were published incorrectly throughout the article. These errors were introduced after the regular proofing process had been completed. The publisher regrets the error and extends its apologies to the author and to the editors and readers of Neophilologus.

The symbol $\beta$ was published incorrectly as Ô. In the footnote on page 217 , the corrected word should read großen.

The symbol $\ddot{a}$ was published incorrectly as $\partial$. In the footnote on page 217 , the corrected word should read Annäherungen.

The symbol $i$ was published incorrectly as $U$. On page 219 , in the penultimate line of the first paragraph, the corrected word should read Lusíadas; on page 219, in the second line of the second paragraph, the corrected word should read Fischart die.

The symbol $\ddot{u}$ was published incorrectly as $\square$. On page 220 , in the penultimate line of the first paragraph, the corrected word should read as frühbürgerliche; on page 226, in the seventh line of the last paragraph, the corrected word should read erwürdigen.

The symbol $\ddot{o}$ was published incorrectly as - On page 222, in the fourth line of the third paragraph, the corrected word should read religiöser; on page 228, the first word should read Mönchshurenkrieg.

Regrettably, these errors have also affected a number of references. The corrected references are provided below:

The original article can be found online at https://doi.org/10.1007/s11061-017-9550-8.

Andrew Weeks

caweeks@ilstu.edu

1 Department of Languages, Literatures, and Culture, Illinois State University, Stevenson Hall 236, Campus Box 430, Normal, IL 61791-4300, USA 
Bebel, H. (1907). Schwänke, Bd. 1, hg. und übers. Albert Wesselski. München und Leipzig: Georg Müller.

de Lagarde, G. (1963). La Naissance de l'esprit laïque, 5 (Guillaume d'Ockham, critique des structures ecclésiales). Louvain: Éditions Nauwelaerts.

Dingel, I. (1996). Concordia controversa. Die öffentliche Diskussion um das lutherische Konkordienwerk am Ende des 16. Jahrhunderts. In Quellen und Forschungen zur Reformationsgeschichte 63. Gütersloh: Gütersloher Verlagshaus.

Flacius Illyricus, M. (1552). Wider die Götter in Preussen. Das nur eine einige wesentliche gerechtigkeit Gottes sey/die nemlich/so in den Zehen gebotten offenbaret ist (Magdeburg). Cf. Wider die Götter in Preussen. In Controversia et Confessio Digital, hg. Irene Dingel, http://www.controversia-et-confessio.de/id/f1476 35e-7db9-48a6-b75e-43b4f1444881. Zugang am 6. April 2017.

Flacius Illyricus, M. (undatiert). Verlegung des Warhafftigen vngegründten berichts Hansen Funckens/von der Osiandrischen Schwermerei (ohne Ort, Verlag oder Datum); vgl. Gegen den unbegründeten Bericht von Hans Funck. In Controversia et Confessio Digital, hg. v. Irene Dingel. http://www.controversia-et-confe ssio.de/id/fbede997-80ca-4e6a-909b-405c209fcf4d. Zugang am 6. April 2017); vgl. Matthias Flacius Illyricus, Verlegung des Warhafftigen vngegründten berichts Hansen Funckens/von der Osiandrischen Schwermerei (Magdeburg 1554), Auf der letzten Seite donnert er ,widder den hundtarzt/seine Propheten vnd Götter/die so meisterlich von der inwonung Gottes schwermen“.

Flacius Illyricus, M. [Carolus Azaria Gotsburgensis] (1549) [Magdeburg] 1549, s. Wider den schnöden Teufel. In Controversia et Confessio Digital, hg. Irene Dingel http://www.controversia-et-confessio.de/id/f0a1a136-d6a8-4d40-8f3b-d2823 51a7f82. Zugang am 6. April 2017.

Fligge, J. R. (1972). Herzog Albrecht von Preußen und der Osiandrismus, 15221568. Bonn: Inaugural-Dissertation.

Goertz, H.-J. (1987). Pfaffenhaß und groß Geschrei: Die reformatorischen Bewegungen in Deutschland, 1517-1529. München: Beck.

Janssen, J. (1883). Ein zweites Wort an meine Kritiker, nebst Ergänzungen und Erläuterungen zu den drei ersten Bänden meiner Geschichte des deutschen Volkes. Freiburg im Breisgau: Herder.

Lemnius, S. (1919). Monachopornomachia (Der Mönchshurenkrieg), hg. G. Vorberg. München: Goerg Müller Verlag.

Manuel, N. (1525). Die Ablaßkrämer. In Altdeutsche Übungstexte 17. Munich: Francke, 1960.

Müller, J.-D. (1984). Curiositas und Erfahrung der Welt im frühen deutschen Prosaroman. In Literatur und Laienbildung im Spätmittelalter und in der Reformationszeit, hg. Ludger Grenzmann and Karl Stackmann. Stuttgart: Metzler.

Müller, J.-D. (April 2014). James M. van der Laan/Andrew Weeks (Hg.), The Faustian Century: German Literature and Culture in the Age of Luther and Faustus. - Günther Mahal, Johann Georg Faustus (1478-1541): Annäherungen an einen großen Unbekannten. In Arbitrium: Zeitschrift für Rezensionen zur germanistischen Literaturwissenschaft (32:1). 
Müller, H., \& Stürmer, M. (1982). Veröffentlichte Referate. In Deutscher Sonderweg-Mythos oder Realität?. München: Kolloquien des Instituts für Zeitgeschichte.

Pierus, U. (1970). Geschichte der kursächsischen Kirchen- und Schulreformation, hg. Thomas Klein. Marburg: Elwert.

Price, D. (2007). Frischlin, N. Phasma (Erstaufführung Tübingen, 1580; Erstdruck Strassburg, 1592). Sämtliche Werke. Dramen, Bd. 3, Teil 2. In Berliner Ausgaben. Sektion philologische Wissenschaften. Stuttgart-Bad Cannstatt: Frommann-Holzboog.

Theatrum Diabolorum, Das ist: Warhaffte, eigentliche vnd kurtze Beschreibung/Allerley grewlicher/schrecklicher vnd abschewlicher Laster/so in diesen letzten/schweren vnd bösen Zeiten/an allen orten vnd enden fast bräuchlich/auch grausamlich im schwang gehen/Darau $\beta$ ein jeder frommer Christ sonderlich zusehen vnd fleissig zu lernen/wie daß wir in disem elenden vnd müheseligen Leben/nit mit Keysern/Königen/Fürsten vnd Herrn/oder anderen hohen gewaltigen Potentaten/sondern mit dem aller mächtigsten vnd stärcksten Fürsten dieser Welt/dem Teufel/zu kämpfen und zustreiten etc. (zitiert als TD 1575). Frankfurt am Main: Peter Schmid.

Tolley, B. (1995). Pastors and Parishioners in Württemberg during the Late Reformation, 1581-1621. Stanford: Stanford UP.

von Schade, H. (Ed.) (1981). Joachim Westphal und Peter Braubach: Briefwechsel zwischen dem Hamburger Hauptpastor, seinem Drucker-Verleger und ihrem Freund Hartmann Beyer in Frankfurt am Main über die Lage der Kirche und die Verbreitung von Büchern. Arbeiten zur Kirchengeschichte Hamburgs 15, hg. Martin Elze, Bernhard Lohse and Hans-Otto Wölber. Hamburg: Wittig Verlag. 